\title{
Long wavelength bifurcation of gravity waves on deep water
}

\author{
By P. G. SAFFMAN \\ Applied Mathematics, California Institute of Technology, \\ Pasadena, California 91125
}

(Received 12 December 1979 and in revised form 24 April 1980)

Conditions are found for the appearance of non-uniform progressive waves of permanent form from a long-wave modulation of a finite-amplitude Stokes wave on deep water. The waveheight at which the modulated waves can occur is a very slowly decreasing function of the modulation wavelength for values up to 150 times the original wavelength. Some qualitative remarks are made about the problem of determining the stability of the new waves.

\section{Introduction}

In a recent paper, Chen \& Saffman (1980) reported the discovery that steady irrotational gravity waves of sufficiently large amplitude are not unique and can bifurcate subharmonically into a new type of wave of permanent form in which crests or troughs have unequal levels. Two cases were examined in detail, in which the wavelength of steady solutions could double or triple at the critical values $h / \lambda=0.1289$ and $0 \cdot 1288$ respectively. Here $h$ denotes the wave height (crest to trough) and $\lambda$ the wavelength of the uniform wave train. At the critical waveheights, new branches of steady solutions exist in which the crests or troughs are not all the same height and the wave is not symmetrical about all the crests and troughs. It is interesting that Garabedian (1965) proved by symmetrization of a variational formulation that water waves with equal crests and troughs and given wave height are unique and symmetrical, and comments that he was unable to prove uniqueness if the possibility that the waves might have crests or troughs of unequal heights was not excluded.

In the present paper, we propose to extend the study of the bifurcation phenomena to include subharmonic bifurcation of arbitrary wavelength relative to the uniform wave train. It should perhaps be emphasized that we are not calculating the unsteady development or the stability of a subharmonic disturbance to a uniform wave train. Steady waves of permanent form and given spatial peried form families or branches which depend continuously upon a parameter such as wave height or energy or wave speed. There may be more than one steady wave for some values of the parameter. We study here the finite-amplitude bifurcation associated with the meeting of two (or more) branches and the appearance of new types of waves.

It is, of course, possible and indeed likely that the stability properties of the branches change at the bifurcation point, and this is a matter of considerable interest. However, our approach to the computational problem makes it convenient to deal with the bifurcation and the changes in stability in ways very different in detail, and we shall therefore postpone a quantitative calculation of stability to later work, and confine ourselves here to a qualitative discussion to be given in $\S 5$. 


\section{Formulation}

It can be shown that the surface of a steady progressive irrotational symmetrical gravity wave on deep water can be expressed parametrically as

$$
X=\xi+\sum_{1}^{\infty} \frac{A_{n}}{n} \sin n \xi, \quad Y=\frac{1}{2} A_{0}+\sum_{1}^{\infty} \frac{A_{n}}{n} \cos n \xi
$$

(see, for example, Schwartz 1974). The wave is symmetric about $\xi=0$ and $\xi=\pi$. We choose units of length and time so that the length of the window in which the waves are observed is $2 \pi$ and $g=1$. The axes are supposed to move with the speed, $c$, of the wave and the origin is taken about a crest or trough, about which the wave is symmetrical, at a level such that the Bernoulli equation expressing constant pressure on the free surface is

$$
q^{2}=-2 Y
$$

where $q$, the speed of the water at the surface, is given by

$$
\frac{c^{2}}{q^{2}}=\left(\frac{d X}{d \xi}\right)^{2}+\left(\frac{d Y}{d \xi}\right)^{2}
$$

Substitution of (2.1) into (2.3) and (2.2), and the comparison of the coefficients of $\cos n \xi, n=0,1,2, \ldots$, gives an infinity of equations in the $\infty+1$ unknowns $c, A_{0}, A_{1}, A_{2}$, .... These equations (they were used by Stokes 1880) are cubic in the unknowns. They are sufficient for our purpose (and they were in fact employed to provide a check on the results), but it turns out to be more convenient to use a quadratic set recently presented by Longuet-Higgins (1978a). He discovered that for steady waves on deep water

$$
\int_{0}^{2 \pi} e^{i m \xi} Y\left(\frac{d X}{d \xi}+\frac{i d Y}{d \xi}\right) d \xi=-\pi c^{2} \delta_{0 m}
$$

for $m=0,1,2,3, \ldots$ Substitution of the forms (2.1) into (2.4) and evaluation of the integrals gives an infinity of quadratic equations for the unknowns

$$
\begin{gathered}
A_{0}+\sum_{1}^{\infty} \frac{A_{n}^{2}}{n}=-c^{2}, \\
A_{l}\left(A_{0}+\frac{1}{l}\right)+\sum_{q=1}^{l-1} \frac{A_{q} A_{l-q}}{q}+\sum_{q=1}^{\infty} \frac{A_{q} A_{l+q}}{q}=0,1 \leqslant l<\infty .
\end{gathered}
$$

(The notation here differs from that used by Longuet-Higgins 1978a.)

The remainder of the paper is concerned mainly with properties of the solutions of the system (2.6). Equation (2.5) can be regarded as giving the wave speed $c$ after the Fourier coefficients have been determined as solutions of (2.6).

\section{Regular waves}

We first used the system (2.6) to calculate the Fourier coefficients $A_{n}$ of regular (uniform) waves of wavelength $\lambda=2 \pi$ for values of $h / \lambda \leqslant 0 \cdot 13173$. The first $L_{1}$ equations of (2.6) were retained, and we put $A_{l}=0$ for $l>L_{1}$. The system of $L_{1}$ quadratic equations in the $L_{1}+1$ unknowns $A_{0}, A_{1}, \ldots, A_{L_{1}}$ was solved by Newton's 
method for given values of one coefficient. Either $A_{1}$ or $A_{2}$ was used to fix the wave amplitude, since the Fourier coefficients are not monotonic functions of the wave height (Schwartz 1974). Thus continuation in just one of the coefficients would run into trouble at the wave height for which it attains a maximum; but this height is different for each Fourier coefficient and switching the given coefficient avoids the difficulty. The computations were carried out on the STAR 100 computer. The chosen value for $L_{1}$ was usually 300 . For the highest wave that we calculated, $A_{300}$ was $O\left(10^{-8}\right)$. Some of the calculations were repeated with $L_{1}=500$; differences in the first 300 Fourier coefficients were less than $10^{-9}$. The kinetic and potential energies per unit area can be expressed as sums over the Fourier coefficients. The values calculated agreed to at least five significant figures with those given, for example, by Cokelet (1977) or Chen \& Saffman (1980). The maximum value of $A_{1}$ was calculated to be $0 \cdot 29721$ for $h / \lambda=0 \cdot 1309$, in agreement with the value given by Schwartz (1974). The maximum value of $A_{2}$ occurs for a wave whose height is greater than those calculated here.

We now consider the lack of uniqueness of solutions of the system (2.6). Suppose that the set $\left\{\bar{A}_{n}\right\}$ constitutes a solution of the system for a wave of given slope $h / 2 \pi$ and wavelength $2 \pi$ with a crest at $\xi=0$, then the set $\left\{B_{n}^{(N)}\right\}$ is also a solution where $N$ is a fixed integer and the $B_{n}^{(N)}$ are defined by

$$
\left.\begin{array}{l}
B_{0}^{(N)}=\bar{A}_{0} / N, \quad B_{p N}^{(N)}=\bar{A}_{p} \quad(p=1,2,3, \ldots), \\
B_{n}^{(N)}=0 \quad \text { otherwise. }
\end{array}\right\}
$$

The wave described by (3.1) has the same slope but its wavelength is now $2 \pi / N$ and its height and speed squared are decreased by the factor $1 / N$. Our terminology is to call this solution a regular wave of class $N$ for our system.

This lack of uniqueness of solutions of (2.6) expressed by (3.1) is, of course, trivial. In mathematical terms, it is a consequence of the infinite degeneracy of the bifurcation of a flat surface into infinitesimal progressive waves, the so-called primary bifurcation. Physically, it corresponds to the similarity of gravity waves of the same slope and different wavelength. Consequently, it appears on first sight that in calculating finite amplitude water waves by solving systems like (2.6), which imposes a spatial period $2 \pi$ on the solution, it suffices to study only the class $N=1$ for which the spatial period or length of the window is the same as the wavelength (this being defined as the distance between identical neighbouring crests). The other solutions of (2.6), for the classes $N>1$, should just describe the same waves with a change of scale. This is true, but Chen \& Saffman (1980) discovered that the solutions for classes $N=2$ and $N=3$ have another feature; namely, at certain critical amplitudes the system has a secondary bifurcation and there exist solutions which are continuous developments of (3.1) but $B_{1}^{(2)}$ and $B_{1}^{(3)}$ are non-zero. In our formulation, this means that a wave of wavelength $2 \pi / 2$ or $2 \pi / 3$ can at certain amplitudes acquire subharmonic components of period $2 \pi$ and become a new type of wave of permanent form, which is not similar to a wave of class 1. Mathematical reasons made it convenient to work with this formulation in which $2 \pi$ is the spatial period of the modulated solutions and the regular (uniform) wavetrain has wavelength $2 \pi / N$. But by an appropriate change of scale, we can of course describe the new solutions found by Chen \& Saffman as a subharmonic bifurcation or modulation of a uniform wavetrain of arbitrary wavelength 
$\lambda$ by components with double or triple the wavelength at critical values of the slope $h / \lambda$. The new waves appear to exist only when the wave is steep with height within $9 \%$ of the sharp peaked limiting wave $(h / \lambda=0 \cdot 141)$. The calculation method actually employed by Chen \& Saffman used a different formulation from the system (2.6), which allowed the calculation of waves with greater height than can be studied with reasonable values of $L_{1}$ since the Fourier coefficients decay too slowly as the wave height approaches the maximum. They were able to follow the bifurcated branches until they almost peaked. However, the approach was limited to small $N$ as the cost increased rapidly with $N$.

In the present paper, we shall describe a direct approach to the calculation of the secondary bifurcation, which is valid for the wave classes with larger $N$. This corresponds to finding waves of permanent form which are combinations of a uniform wave train of wavelength $\lambda$ and subharmonic components of wavelength up to $N \lambda$. The method does, however, have the drawback that it cannot be used to follow the new branches for finite distances.

One further point needs to be made about the uniqueness of regular waves. In addition to the wave described by (3.1), the set

$$
B_{0}^{(N)}=\bar{A}_{0} / N, \quad B_{p N}^{(N)}=(-1)^{p} \bar{A}_{p}
$$

is also a regular wave of class $N$ symmetrical about the origin. The difference between (3.1) and (3.2) is just a horizontal displacement $\frac{1}{2} \lambda=\pi / N$ so that crests and troughs are interchanged. This degeneracy, which is due to invariance under translation, may again seem trivial but must be considered when $N$ is even. The reason is that the representation (2.1) restricts us to waves which are symmetrical about $\xi=0$ and $\xi=\pi$. Waves of odd class have a crest at $\xi=0$ and a trough at $\xi=\pi$ (or vice versa) and hence bifurcated branches will be symmetrical about at least one crest and one trough. It is in this case sufficient to limit attention to regular waves that have a crest at $\xi=0$ and correspondingly have $B_{p N}^{(N)}>0$.

But waves of even class with $B_{p N}^{(N)}>0$ have a crest at both $\xi=0$ and $\xi=\pi$. The bifurcated branches of regular waves with $N$ even and $B_{N}^{(N)}>0$ are therefore symmetrical about two crests but not about any troughs. Bifurcations with $N$ even and $B_{N}^{(N)}<0$ are different waves and will be symmetrical about two troughs but not about any crests. It is not obvious that the critical waves are the same for each class, but it will in fact appear that for $N \geqslant 4$ the two bifurcations appear together; more than one new branch therefore occurs at the same slope. For $N=2$, bifurcation is found only for waves that are symmetrical about crests.

\section{The bifurcation criterion}

A necessary condition for the bifurcation of a regular wave of class $N$ into a symmetrical irregular wave is found by substituting

$$
A_{0}=B_{0}^{(N)}, \quad A_{n}=B_{n}^{(N)}+\epsilon A_{n}^{\prime} \quad(n>0)
$$

into (2.6), and asking if the homogeneous linear equations for the $A_{n}^{\prime}$, obtained by equating the coefficients of $\epsilon$ to zero, have non-trivial solutions. The vanishing of the determinant of the system of linear equations gives the amplitude at which a regular 
wave of class $N$ might bifurcate. It is convenient to introduce a different notation in order to describe the computational procedure. We write the system (2.6) as

$$
\mathbf{F}(\mathbf{x}, y)=0
$$

where

$$
\mathbf{x}=\left(A_{1}, A_{2}, A_{3}, \ldots\right), \quad y=-A_{0} .
$$

The components $F_{I}(I=1,2,3, \ldots)$ of the vector $\mathbf{F}$ are

$$
F_{I}(\mathbf{x}, y) \equiv\left(\frac{1}{I}-y\right) x_{I}+\sum_{1}^{\infty} \sum_{1}^{\infty} \alpha_{J}^{I} K_{K} x_{J} x_{K}
$$

The coefficients $\alpha_{J, K}^{I}$ are constants with the values

$$
\begin{aligned}
& \alpha_{J, K}^{I}=\frac{1}{2}\left(\frac{1}{J}+\frac{1}{K}\right) \quad \text { if } \quad J+K=I, \\
& =\frac{1}{2 K} \quad \text { if } \quad J-K=I, \\
& \begin{array}{ll}
=\frac{1}{2 J} & \text { if } \quad K-J=I, \\
=0 & \text { otherwise. }
\end{array}
\end{aligned}
$$

The Jacobian matrix has components

$$
\mathbf{F}_{\mathbf{x}} \equiv \frac{\partial F_{I}}{\partial x_{J}}=\left(\frac{1}{I}-y\right) \delta_{I J}+2 \sum_{1}^{\infty} \alpha_{J, K}^{I} x_{K}
$$

A condition for bifurcation is (e.g. Keller 1977)

$$
\begin{aligned}
& \operatorname{det}\left(\mathbf{F}_{\mathbf{x}}\right)=0, \\
& \frac{\partial \mathbf{F}}{\partial y} \in \mathscr{R}\left(\mathbf{F}_{\mathbf{x}}\right),
\end{aligned}
$$

where $\mathscr{R}$ stands for range. We make the substitution

$$
x_{K}=B_{K}^{(N)}, \quad y=-B_{0}^{(N)}
$$

where the $B$ 's are given by (3.1) or (3.2) and find the critical wave for which bifurcation occurs by finding the wave of class $N$ for which (4.7) is satisfied, and then verifying (4.8).

It is clear from inspection that for $N \geqslant 3$ the Jacobian matrix can be factored into independent blocks, where each block consists of elements from the rows and columns

$$
I=s N \pm p, \quad J=s N \pm p
$$

where $s$ is a positive integer or zero, and $p$ is a positive integer which can be taken to be prime relative to $N$ and not greater than $\frac{1}{2} N$, without loss of generality. The corresponding eigenvector describes an infinitesimal perturbation with non-zero Fourier coefficients $A_{p}^{\prime}, A_{N-p}^{\prime}, A_{N+p}^{\prime}, A_{2 N-p}^{\prime}, A_{2 N+p}^{\prime}$, etc. to the regular wave with wavelength $2 \pi / N$. If $p=1$, the bifurcation can be regarded as an $N$-fold increase in wavelength associated with the appearance of sidebands of wavenumber $m N \pm 1$ about the harmonics of wavenumber $m N$ of the uniform wave. 
Each block is a matrix $\mathbf{G}$, where

and

$$
G_{i j}=\partial F_{I} / \partial x_{J}
$$

$$
\left.\begin{array}{lll}
I=p & \text { if } \quad i=1, \\
I=(i-1) N-p & \text { if } i=2,4,6, \ldots, \\
I=(i-1) N+p & \text { if } \quad i=3,5,7, \ldots, \\
J=p & \text { if } j=1, \\
J=(j-1) N-p & \text { if } j=2,4,6, \ldots, \\
J=(j-1) N+p & \text { if } j=3,5,7, \ldots
\end{array}\right\}
$$

The matrix $\mathbf{G}$ was truncated to the first $L_{2}$ rows and columns, and we put $\bar{A}_{n}=0$ for $n>L_{1}$. The values of $\operatorname{det}(\mathbf{G})$ were calculated as functions of $h / \lambda$ for given $N$ and $p$. In practice, the regular wave was characterized by the value of $\bar{A}_{2}$. The size of $L_{2}$ was usually 401 but some results were repeated with $L_{2}=701$ to check the accuracy of the calculations. It took about $2 \mathrm{~s}$ on the CDC STAR 100 to calculate the determinant of the $401 \times 401$ matrix. Mullers method of finding the roots of a function was employed to find the value of $\bar{A}_{2}$ for which the determinant vanished. The zero was supposed found when the value $\bar{A}_{2}$ in the next iteration differed from the previous one by less than $5 \times 10^{-7}$. For each $\bar{A}_{2}$, it took about $2 \mathrm{~s}$ to calculate 300 Fourier coefficients and the properties of the regular wave. (It proved convenient to normalize G by multiplying each column, other than the first, by $N$ before calculating the determinant and the eigenvector of the singular matrix.)

Because of the structure of (4.6) and (4.12), it is clear that the transformation of (3.1) into (3.2) multiplies each element of $\mathbf{G}$ by $(-1)^{i+j}$ and therefore leaves $\operatorname{det}(\mathbf{G})$ unaltered. Thus for $N \geqslant 3$, bifurcation occurs at the same height of the regular wave irrespective of whether the bifurcation is crest or trough symmetrical. For $N$ odd, the bifurcated waves are the same, since they differ only by a translation, but for $N$ even they are physically distinct. Nevertheless, it is sufficient as far as the necessary condition for bifurcation is concerned to limit attention to crest symmetry.

The case $N=2$ is special. Now the Jacobian (4.6) breaks up into independent blocks formed out of the odd and even rows. The odd rows give the determinant whose vanishing is a necessary condition for subharmonic bifurcation, and the corresponding eigenvector gives the perturbation $A_{1}^{\prime}, A_{3}^{\prime}, A_{5}^{\prime}, \ldots$ to the regular wave of class 2 . The elements of the matrix $\mathbf{G}$ are now for crest symmetry (3.1)

$$
\left.\begin{array}{rl}
G_{i i} & =\frac{\bar{A}_{0}}{2}+\frac{1}{2 i-1}+\frac{\bar{A}_{2 i-1}}{2 i-1}, \\
G_{i j} & =\frac{\bar{A}_{j-i}}{2(j-i)}+\frac{\bar{A}_{j+i-1}}{2 j-1} \text { if } i<j, \\
G_{i j} & =\frac{\bar{A}_{i+j-1}}{2 j-1}+\bar{A}_{j-i}\left(\frac{1}{2 j-1}+\frac{1}{2(i-j)}\right) \text { if } i>j .
\end{array}\right\}
$$

The trough symmetry matrix is obtained from (4.13) by replacing $\bar{A}_{k}$ with $(-1)^{k} \bar{A}_{k}$. It is clear that there is now a significant difference in the matrix $\mathbf{G}$.

The properties of the critical waves of smallest height are listed in table 1 for various values of $N$ up to 150 . The critical waves are the ones for which $\operatorname{det}(\mathbf{G})$ vanishes. 


$\begin{array}{rlcccc}N & h / \lambda & 4 \pi^{2} T / g \lambda^{2} & 4 \pi^{2} V / g \lambda^{2} & 2 \pi c^{2} / g \lambda \\ 2 & \text { crest } & 0.1289 & 0.03711 & 0.03395 & 1 \cdot 175 \\ 2 & \text { trough } & +0.1317 & - & - & -175 \\ 3 & 0.1288 & 0.03708 & 0.03393 & 1 \cdot 175 \\ 4 & 0.1287 & 0.03704 & 0.03390 & 1 \cdot 175 \\ 5 & 0.1286 & 0.03700 & 0.03387 & 1 \cdot 174 \\ 10 & 0.1284 & 0.03691 & 0.03381 & 1 \cdot 174 \\ 20 & 0.1282 & 0.03686 & 0.03376 & 1 \cdot 173 \\ 50 & 0.1281 & 0.03682 & 0.03373 & 1 \cdot 173 \\ 100 & 0.1281 & 0.03680 & 0.03372 & 1.173 \\ 150 & 0.1280 & 0.03680 & 0.03372 & \end{array}$

TABre 1. Properties of smallest critical waves of class $N . c$ is the wave speed, $V$ is the potential energy per unit area, $T$ is the kinetic energy per unit area.

They were found by monitoring the determinant as the wave height was increased from zero and applying Muller's method to find the root more accurately after a change of sign was detected. All the results shown are for $p=1$, corresponding to an $N$-fold increase in wavelength. It was found that, for $N>10$, results for $p>1$ were close to those for $p=1$ and wave class close to $N / p$ (remember $N$ and $p$ are coprime).

It can be seen that the height of the limiting wave decreases as the class increases, but the change is extremely small, being in at most the third significant figure. For $N=2$, no bifurcation was found that gives waves symmetrical about troughs, at least for values of $h / \lambda<0.1317$. This suggests that a wave of maximum height with sharp crests is unique and symmetrical if all the crests are the same height, as suggested by Garabedian's (1965) arguments.

The eigenvectors of $\mathbf{G}$ when $\operatorname{det}(\mathbf{G})=0$ describe the form of the new waves. For the results presented here, it appeared from the computations that the largest components of the eigenvector were those with wavenumber $N \pm 1$, corresponding to the sidebands of the fundamental having the major influence. Now, it is possible that other real zeros of $\operatorname{det}(\mathbf{G})$ may exist, each associated with bifurcation into new waves, in which the dominant components of the eigenvector are not $A_{N_{ \pm 1}}^{\prime}$ but $A_{m N_{11}}^{\prime}$ where $m>1$.

The modulation associated with the new waves would then be due mainly to the appearance of superharmonic sidebands. Some calculations were carried out for $N=200$ and even larger values of $N$. It was found that in these cases it proved difficult to isolate the first zero of $\operatorname{det}(\mathbf{G})$ and there appeared to be many zeros close together. It is believed that this is due to the appearance of subharmonic modulation of the superharmonics, which is occurring at heights close to that for subharmonic modulation of the fundamental when $N$ is large. This is still an unconfirmed speculation but it is hoped that detailed studies of the wave stability currently in progress will clarify further the nature of the bifurcation for very large $N$. (When the matrix $\mathbf{G}$ is normalized by multiplying each column other than the first by $N$, it has a well defined limit as $N \rightarrow \infty$. The zeros of this limit matrix behaved like those of $\mathbf{G}$ for $N \geqslant 200$.) On the other hand, the limit $N \rightarrow \infty$ of infinitely long wavelength bifurcation may be peculiar, because we shall show $(\S 6)$ that Whitham's evolution equations, valid for infinitely long modulations, do not predict bifurcation.

We did not try to follow the new branches and search for tertiary bifurcation as the 
present method is inadequate for this purpose. We shall, however, discuss in the next section the properties of the new branches (irregular waves) when they are close to the critical wave. Chen \& Saffman followed the irregular waves for $N=2$ and $N=3$ to within $1 \%$ of the highest wave and did not detect tertiary bifurcation in these cases.

\section{Initial properties of the new branches}

We return to the formulation (4.2). Let ${ }^{*}$ denote differentiation along a branch with respect to arc length. Following one of the usual methods (e.g. see Keller 1977), we differentiate (4.2) and evaluate at the bifurcation point, giving

$$
\mathbf{F}_{\mathbf{x}} \dot{\mathbf{x}}=-\mathbf{F}_{y} \dot{y}
$$

The numerical calculations indicated (at least for $N \leqslant 150$ ) that the zero of $\operatorname{det}\left(\mathbf{F}_{\mathrm{x}}\right.$ ) is simple and hence that the null space of $\mathbf{F}_{\mathbf{x}}$ is one-dimensional. Let $\psi(|\psi|=1)$ span the null space, and $\psi^{\dagger}$ denote the adjoint vector. Then the solution of $(5.1)$ is

where $\phi$ is the unique solution of

$$
\dot{\mathbf{x}}=\dot{y} \phi+\alpha \psi
$$

and $\alpha$ is an unknown scalar.

$$
\mathbf{F}_{\mathbf{x}} \boldsymbol{\phi}=-\mathbf{F}_{y},\langle\psi, \phi\rangle=0
$$

The cases $N=2$ and $N=3$ were computed by Chen $\&$ Saffman. Here, we consider the case $N \geqslant 4$. For given wave class $N$ and sideband number $p, \psi$ is a vector whose only non-zero components are those of index $s N \pm p$. This corresponds to the perturbation having Fourier coefficients $A_{s N \pm p}^{\prime}$, see (4.10). The quantity $\psi$ is, of course, just the normalized right eigenvector of $\mathbf{F}_{\mathbf{x}}$ and $\psi^{+}$is the left eigenvector (transposed). The only non-zero components of $\phi$ are those with index $s N$. In fact

evaluated for the critical wave.

$$
\phi_{s N}=N\left(\partial \bar{A}_{s} / \partial \bar{A}_{0}\right)
$$

The ratio of $\alpha$ and $\dot{y}$ is found by taking the second derivative of (4.2) and forming the inner product with $\psi^{+}$, which gives

$$
\dot{y}^{2}\left\langle\psi^{+}, \mathbf{F}_{\mathbf{x x}} \boldsymbol{\phi} \phi+\mathbf{F}_{y y}+2 \mathbf{F}_{\mathbf{x} y} \boldsymbol{\phi}\right\rangle+2 \dot{y} \alpha\left\langle\psi^{+}, \mathbf{F}_{x \mathbf{x}} \phi \psi+\mathbf{F}_{x y} \psi\right\rangle+\alpha^{2}\left\langle\psi^{+}, \mathbf{F}_{x x} \psi \psi\right\rangle=0
$$

Remembering that $\mathbf{F}$ is given by (4.4), we have

Since

$$
\left(\mathbf{F}_{\mathrm{xx}} \psi \psi\right)_{i}=2 \sum_{j} \sum_{k} \alpha_{j k}^{i} \psi_{j} \psi_{k}
$$

$$
N \pm p \neq\left(s^{\prime} N \pm p\right)+\left(s^{\prime \prime} N \pm p\right)
$$

for $N \geqslant 4$ when $p<\frac{1}{2} N$, it is easily verified from the definition of $\alpha_{j k}^{i}$ and the properties of $\psi$ that the last term in (5.5) vanishes. The first term in (5.5) also vanishes, because $\alpha=0$ must be a solution corresponding to continuation along the regular branch. We assume that the middle term in (5.5) does not vanish, as can be verified by computation. Then

$$
\dot{\mathbf{x}}=\psi, \quad \dot{y}=0,
$$

along the bifurcating branches $(\alpha=1$ without loss of generality by suitable choice of arc length), which are therefore given to leading order by

$$
\mathbf{x}=\mathbf{x}_{0}+\epsilon \psi+O\left(\epsilon^{2}\right), \quad y=y_{0}+O\left(\epsilon^{2}\right)
$$


where $\mathrm{x}_{0}, y_{0}$ is the bifurcation point. Now $\mathbf{x}_{0}$ and $\psi$ are orthogonal, and hence the wave speed $c$ is constant to $O(\epsilon)$ along the bifurcated branch. Thus to first order, the new branches are characterized by the appearance of the Fourier components $A_{s N \pm p}$ and an unaltered wave speed. (Note that the condition (5.7) can be violated if $N=3$ and $p=1$. Thus (5.8) is not necessarily true on the new branch for $N=3$ and in fact the calculations by Chen \& Saffman for the $N=3$ bifurcation showed that $\dot{c} \neq 0$ on the bifurcation branches in this case.)

The procedure for calculating to $O\left(\epsilon^{2}\right)$ on the bifurcating branch is similar. We now differentiate (4.2) twice, evaluate at the bifurcation point and substitute (5.8) to give

$$
\mathbf{F}_{\mathrm{x}} \ddot{\mathbf{x}}=-\mathbf{F}_{y} \ddot{y}-\mathbf{F}_{\mathrm{xx}} \psi \psi \text {. }
$$

Equation (5.5) was just the condition that the right-hand side lies in the range of $\mathbf{F}_{\mathbf{x}}$. Then

$$
\ddot{\mathbf{x}}=\ddot{y} \phi+\mathbf{F}_{\mathbf{x}}^{-1} F_{\mathbf{x x}} \psi \psi
$$

where $\mathbf{F}_{\mathbf{x}}^{-1}$ denotes the unique inverse operator which gives a vector orthogonal to $\psi$. The definition of arc length by the equations

$$
|\dot{\mathbf{x}}|^{2}+\dot{y}^{2}=1, \quad\langle\dot{\mathbf{x}}, \ddot{\mathbf{x}}\rangle+\dot{y} \ddot{y}=0,
$$

ensures that the arbitrary multiple of $\psi$ which could be added to (5.11) vanishes. The second term in (5.11) produces Fourier coefficients $A_{s N}^{\prime}$ and $A_{s N \pm 2 p}^{\prime}$. It remains to find $\ddot{y}$. For this purpose, take the third derivative of (4.2), evaluate at the bifurcation point, substitute (5.8) and (5.11), and form the inner product with $\psi^{+}$to eliminate $\ddot{\mathbf{x}}$ and $\dddot{y}$. The result is

$$
\ddot{y}=-\left\langle\psi^{\dagger}, \mathbf{F}_{\mathbf{x x}} \psi \chi x+\frac{1}{3} \mathbf{F}_{\mathbf{x x}} \psi^{3}\right\rangle /\left\langle\psi^{\dagger}, \mathbf{F}_{\mathbf{x} y} \psi+\mathbf{F}_{\mathbf{x x}} \phi \psi\right\rangle,
$$

where $\chi$ is the second member of the right-hand side of (5.11). The actual values have not yet been computed. The second order of change in wave speed will follow from (2.5).

Some preliminary qualitative remarks on the problem of computing the changes in stability at a bifurcation are appropriate as the calculation is likely to be somewhat difficult. Suppose the surface is described by a real state vector $u$ relative to a coordinate system moving with a speed $C$ by a nonlinear real equation

$$
u_{i}+M(u, C)=0 \text {. }
$$

It is to be noted that $u$ cannot be restricted to symmetrical states, which (denoted above by $\mathbf{x}$ ) are only a subspace of $u$. An initially symmetrical surface on a current will immediately lose its symmetry unless it happens to be a steady progressive wave. (We drop the use of boldface to emphasize the difference between the general vector $u$ and the particular symmetric state vector $\mathrm{x}$ of $\S 4$.)

Suppose that $u=w, C=c$ denotes a steady progressive wave, so that

$$
M(w, c)=0 .
$$

The stability to infinitesimal perturbations $v e^{\sigma t}$ of this steady solution is determined by the eigenvalues $\sigma$ of the linear eigenvalue problem for the vector $v$,

$$
M_{u}(w, c) v+\sigma v=0
$$

where $M_{u}$ denotes the Fréchet derivative. The existence of an eigenvalue $\sigma$ such that $\operatorname{Re}(\sigma)>0$ implies instability. If $\operatorname{Re}(\sigma) \leqslant 0$ for all eigenvalues, the wave is said to be 
stable. If $\sigma=0$ for some eigenvector, the disturbance is neutrally stable. Note that $\sigma=0$ will always be an eigenvalue because the waves are invariant under translation and hence the rate of change of $w$ with respect to horizontal displacement will be a null eigenvector. Thus $M_{u}$ is in this sense always singular. Our previous calculations of steady waves avoided this difficulty by limiting attention to symmetrical solutions, which this eigenvector is obviously not. However, the interest here lies in the eigenvalues which just pass through zero for a particular waveheight, for which the corresponding eigenvector is neutrally stable, and bifurcation to a new branch of steady waves may occur. The trivial singularity or bifurcation associated with the translation invariance is not significant, but it can complicate numerical investigations because the singularity of $M_{u}$ at a non-trivial bifurcation is not of first order.

Since water waves are non dissipative and the flow is reversible, it follows that $-\sigma$ is also an eigenvalue. In addition, $\sigma^{*}$ and $v^{*}$ are an eigenvalue and eigenvector since $M_{u}$ is real. Thus $\sigma$ is pure imaginary for stable disturbances which come in pairs, whereas if $\sigma$ is complex and the wave is unstable, the disturbances come in quartets unless $\sigma$ is real and they again come in pairs. A neutrally stable disturbance is therefore associated with a double or quadruple zero. (The zero corresponding to the trivial displacement is ignored.)

Let us suppose we follow a stable solution branch up to and through a point of neutral stability, where $\sigma$ has a double zero. There may or may not be an exchange of stability on this branch. If there is a change of stability, $\sigma$ changes from pure imaginary to complex. This requires non-analytic behaviour. If the number of independent eigenvectors of $M_{u}$ does not alter, $\sigma$ is analytic and hence there cannot be a change of stability. If a change of stability occurs, the two eigenvectors must coalesce at the critical point, and the dimension of the null space of $M_{u}$ is one.

A change of stability can, of course, occur away from a point of neutral stability, provided that two eigenvalues coincide and the eigenvectors coalesce. Equality of the eigenvalues without coalescence of the eigenvectors will mean that $\sigma$ is analytic and cannot change from pure imaginary to complex.

These ideas are illustrated in the sketch of figure 1, where we show the imaginary part of eigenvalues, corresponding to disturbances composed of components of wave numbers $(s N+p),-\infty<s<\infty$, to a regular wave of class $N$, as functions of $h / \lambda$. For $h / \lambda=0$, the eigenvalues are pure imaginary and given by

$$
\operatorname{Im} \sigma=\frac{m N+p}{\sqrt{N}} \pm|m N+p|^{\frac{1}{2}}
$$

where $m$ is an arbitrary integer (positive, negative or zero), determined by the dominant component of the eigenvector. The first term in (5.17) is due to convection with the speed $1 / \sqrt{ } N$ of the co-ordinate system. The second term describes the propagation of the free wave relative to the water; the radical is positive if the disturbance is coflowing and negative if it is counter-flowing.

The behaviour shown in figure 1 described by $A B C$ occurs for $N=2, p=1, m= \pm 1$, according to the computations of Longuet-Higgins (1978b). The behaviour $A B D$ appears to describe the phenomena with $N>2, m= \pm 1, p \neq \frac{1}{2} N$. There are as yet no published calculations for $|m|>1$, but our numerical results suggest that behaviour $E F$ is possible in this case. There will clearly be coincidence of eigenvalues occurring many times, but this will not in general produce transition. When $N \gg 1$, the value of 


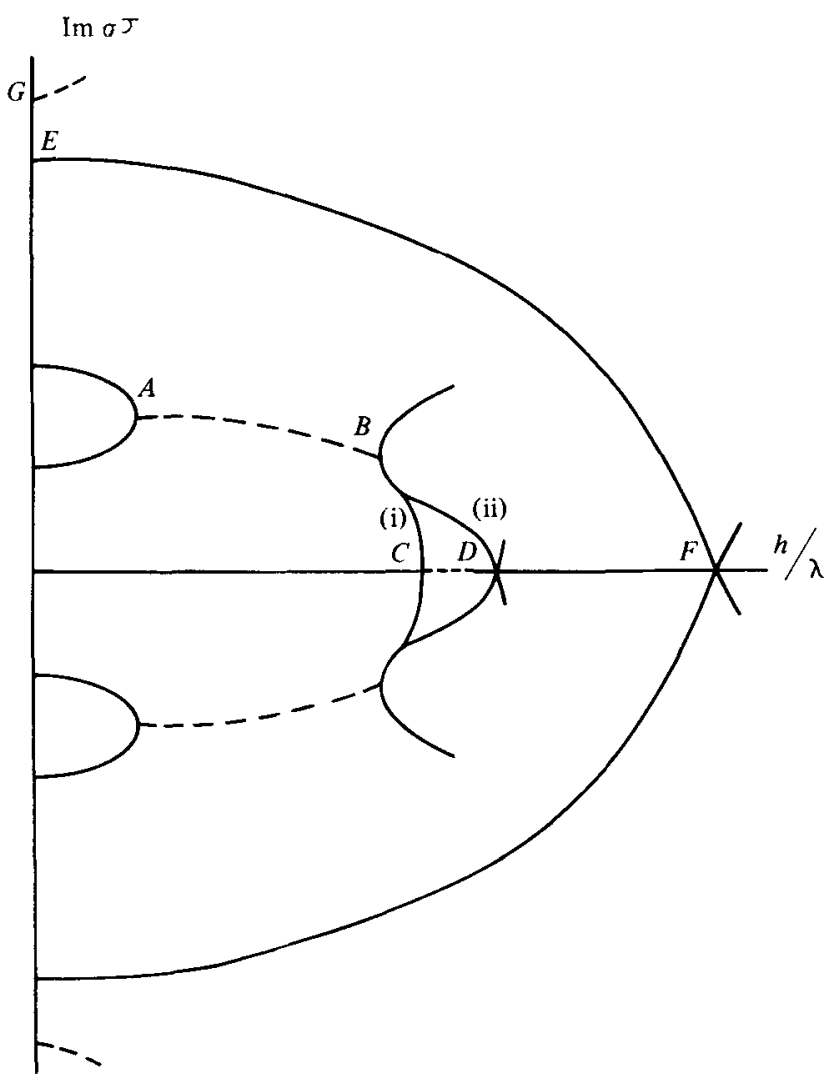

Figure 1. Possible behaviour of $\operatorname{Im} \sigma$ for eigenvalues associated with disturbances of wavenumber $\pm(s N+p)$ to a regular wave of class $N$. The solid lines are stable disturbances, $\operatorname{Re} \sigma=0$, with single eigenvectors. The dashed lines are for two eigenvectors, with equal and opposite values of $\operatorname{Re} \sigma$. Point $A$, coalescence of two eigenvectors and transition to instability; $B$, restabilization and splitting of eigenvalues, with one of the eigenvalues following path $B C$ (i) or alternatively $B D$ (ii); $C$, neutral stability with coalescence of eigenvectors leading to transition, Longuet-Higgins $(1978 b) ; D$, neutral stability without coalescence and no transition; $F$, neutral stability without coalescence for disturbances that are always stable; $G$, possible instability for $h / \lambda>0$ associated with equal eigenvalues when $h / \lambda=0$ (parametric instability). Dashed line from $C$ represents two eigenvectors with $\sigma$ proportional to $\pm\left\{h / \lambda-(h / \lambda)_{c}\right\}^{\frac{1}{2}}$.

$h / \lambda$ for point $A$ is given by the well-known results for the Benjamin-Feir instability of sidebands of wavenumber $1 \pm p / N$ of a uniform wavetrain of wavenumber unity;

$$
\frac{h}{\lambda}=\frac{p}{2 \sqrt{ } 2 \pi N}
$$

One further point deserves special mention. If eigenvalues coincide for $h / \lambda=0$, the eigenvalue may be complex for $h / \lambda>0$. It follows from (5.17) after a little algebra that a co-flowing perturbation $(N, p)$ with positive mode number $m_{1}$ and a counterflowing disturbance with the same $(N, p)$ and positive mode number $m_{2}$ will have equal values of $\sigma$ if

$$
4 p=N\left\{\left(m_{2}-m_{1}\right)^{2}-2\left(m_{1}+m_{2}\right)+1\right\}
$$


This equation has an infinite number of integer solutions, with

$$
N=4, \quad p=1, \quad m_{1}=M^{2}-M, \quad m_{2}=M^{2}+M,
$$

where $M \geqslant 0$ is an arbitrary integer. If the behaviour of this double eigenvalue is examined by standard perturbation theory for $h / \lambda \ll 1$, it can be complex or pure imaginary depending on the values of certain matrix coefficients. To see this, note that we can without loss of generality suppose that we are examining an eigenvalue problem of the form

$$
L(\epsilon) u=\sigma(\epsilon) u
$$

where $L(0)$ has two distinct eigenvectors $\phi, \psi$ for eigenvalue $\sigma=0$. Here, $\epsilon=h / \lambda \ll 1$. The formal expansion

$$
\begin{aligned}
& L=L(0)+\epsilon L_{1}+\epsilon^{2} L_{2}+\ldots, \\
& u=\alpha \phi+\beta \psi+\epsilon u_{1}+\epsilon^{2} u_{2} \ldots, \\
& \sigma=\epsilon \sigma_{1}+\epsilon^{2} \sigma_{2}+\ldots,
\end{aligned}
$$

will lead to a quadratic for $\sigma_{k}$ if the matrix coefficients $\psi^{\dagger} L_{k} \phi$, etc. are non-zero. Here, $k=2 M$ and an instability proportional to $(h / \lambda)^{2 M}$ (or slower) is possible. $\dagger$ Such behaviour is illustrated by $G$ in figure 1 , and can be called a subharmonic modulation of the superharmonics. Alternatively, it can be regarded as the parametric instability of the plane surface to disturbances of wavelength $4 \lambda /\left(1+4\left[M^{2} \pm M\right]\right)$ in the presence of a uniform wave train of wavelength $\lambda$.

We come now to the question of the stability of the new branches which bifurcate from the regular waves at points such as $C, D$ or $F$ in figure 1 . It is to be remembered that bifurcation must occur provided these points are not limit points, i.e. points of stationary speed $c$, along the branch of regular waves. The behaviour depends on the dimension of the null space of $M_{u}$. At $D$ and $F$, the null space is two dimensional; at $C$ it is one dimensional. (Again, the contribution of translation invariance to the null space is neglected.) Let us consider first the former case. Differentiation along the branch of irregular waves is denoted by a dot. Differentiating (5.16), we get

$$
\dot{M}_{u} v+M_{u} \dot{v}+\sigma \dot{v}+\dot{\sigma} v=0 .
$$

Let $\phi$ and $\psi$ denote the two independent orthonormal eigenvectors which span $M_{u}$ at the bifurcation point. Then

$$
v=\alpha \phi+\beta \psi .
$$

Multiplying (5.20) at the bifurcation point by the orthonormal adjoints $\phi^{\dagger}$ and $\psi^{+}$, and eliminating $\alpha / \beta$, we obtain

$$
\left(\dot{\sigma}-\phi^{\dagger} M_{u} \phi\right)\left(\dot{\sigma}-\psi^{\dagger} \dot{M} \psi\right)=\left(\phi^{\dagger} \dot{M}_{u} \psi\right)\left(\psi^{\dagger} M_{u} \phi\right)
$$

The roots of this quadratic are either real or pure imaginary (because complex roots come in quartets), and the new branch is accordingly stable or unstable.

Now consider the behaviour at $C$ where the eigenvectors coalesce. The null space is one-dimensional, spanned by $\phi$, and further

$$
\phi^{\dagger} \phi=0 \text {. }
$$

† These ideas were developed from discussion with Dr T. Minzoni who had found this be. haviour in similar situations. Preliminary computations carried out with D. Martin and H. C. Yuen indicate that this instability does indeed exist for $M=1$ with a growth rate proportional to $(h / \lambda)^{4}$. 
We cannot assume now that $\dot{\sigma}$ and $v$ are well behaved. Since $\phi$ is in the range of $M_{u}^{c}$ (where the superfix $c$ denotes evaluation at the bifurcation point) we can put

Substitution into (5.20) gives

$$
\dot{v}=\dot{\sigma}\left(M_{u}^{c}\right)^{-1} \phi+\chi .
$$

$$
\sigma \dot{\sigma}\left(M_{u}^{c}\right)^{-1} \phi+\sigma \chi+\sigma \dot{v}=\dot{M}_{u} v+\dot{\sigma} M_{u}\left(M_{u}^{c}\right)^{-1} \phi+M_{u} \chi
$$

We evaluate at the critical point, where $v=\phi$, and see that there is a solution with $\chi$ bounded provided, after multiplying by the adjoint $\phi^{\dagger}$,

$$
(\sigma \dot{\sigma})^{c}=\phi^{+} \dot{M}_{u}^{c} \phi / \phi^{\dagger}\left(M_{u}^{c}\right)^{-1} \phi .
$$

This shows that $\sigma^{2}$ is linear in arc length along the branch, provided the numerator in (5.26) does not vanish. (The same result can be obtained by expanding $\sigma$ and $v$ in powers of (arc length) $)^{\frac{1}{2}}$ and equating coefficients.) There is then an exchange of stability along the branch, as exemplified by the regular branch at the point $C$. However for the case $N=2, p=1$, the new branch is symmetrical about the bifurcation point which cannot then be a point of stability exchange. In this case, the numerator in (5.26) vanishes. It follows that $\sigma$ is linear in arc length. An expansion of this kind leads to a quadratic for $\dot{\sigma}$, and again the matrix coefficients must be evaluated to determine if the new branch is stable or unstable.

The calculation of the various matrix coefficients is a non-trivial task. In practice, it may be easier to solve (5.16) directly for the eigenvalues. Alternatively, it may prove possible to avoid the heavy calculation by exploiting the variational principle (Garabedian 1965). This approach has proved useful for the stability of vortex arrays (Saffman \& Szeto 1980), but the precise method for the present case is still obscure.

\section{Non-existence of bifurcation for $N=\infty$}

We shall now describe an analytical argument that shows the non-existence of bifurcation in the limit $N / p=\infty$. In the limit of infinitely long modulations to a uniform wave train, the variations of amplitude and wavelength are given exactly by Whitham's $(1965,1970,1974)$ theory. There is no restriction that the variations are small, the only requirement is that they are slow.

The modulation equations take their simplest form if wavenumber $k(x, t)$ and action density $I(x, t)$ are used as dependent variables (Whitham 1974, p. 515):

$$
\begin{gathered}
\frac{\partial k}{\partial t}+\frac{\partial \Omega}{\partial k} \frac{\partial k}{\partial x}+\frac{\partial \Omega}{\partial I} \frac{\partial I}{\partial x}=0 \\
\frac{\partial I}{\partial t}+\frac{\partial J}{\partial I} \frac{\partial I}{\partial x}+\frac{\partial J}{\partial k} \frac{\partial k}{\partial x}=0
\end{gathered}
$$

Here $\Omega=\Omega(k, I)$ and $J=J(k, I)$ are the angular frequency and action flux, respectively, of a uniform wave train.

It can be shown either directly (Longuet-Higgins 1975) or from Whitham's average Lagrangian formulation that on deep water

$$
I=\frac{2 T}{\Omega}, \quad J=\frac{3 T-2 V}{k}, \quad \frac{\partial J}{\partial I}=\frac{\partial \Omega}{\partial k}, \quad \frac{\partial E}{\partial I}=\Omega
$$


where $T$ and $V$ are the average kinetic and potential energy densities and $E=T+V$. From dimensional considerations,

$$
\Omega=(g k)^{\frac{1}{2}} \tilde{\Omega}(\beta), \quad T=\frac{g}{k^{2}} \tilde{T}(\beta), \quad V=\frac{g}{k^{2}} \tilde{V}(\beta), \quad \beta=\frac{I k^{\frac{5}{2}}}{g^{\frac{1}{2}}} .
$$

The functions $\widetilde{\Omega}, \widetilde{T}$ and $\tilde{V}$ can be deduced from the tabulated values (e.g. Cokelet 1977) of $\Omega, T, V$ and $I$ as functions of amplitude.

Consider the infinitesimal perturbation of a uniform wave train. Put

$$
\begin{aligned}
& k=k_{0}+\left\{a_{1} \cos \kappa\left(x-c_{0} t\right)+b_{1} \sin \kappa\left(x-c_{0} t\right)\right\} e^{\sigma t}, \\
& I=I_{0}+\left\{a_{2} \cos \kappa\left(x-c_{0} t\right)+b_{2} \sin \kappa\left(x-c_{0} t\right)\right\} e^{\sigma t} .
\end{aligned}
$$

In terms of the Fourier component representation of $\S 3$, it corresponds to a perturbation with components of index $N \pm N^{2} \kappa$ where $N=1 / k_{0}$. Here $c_{0}=\Omega_{0} / k_{0}$ is the phase speed of the undisturbed regular wave. The eigenvalue problem obtained from linearizing (6.1) and (6.2) is

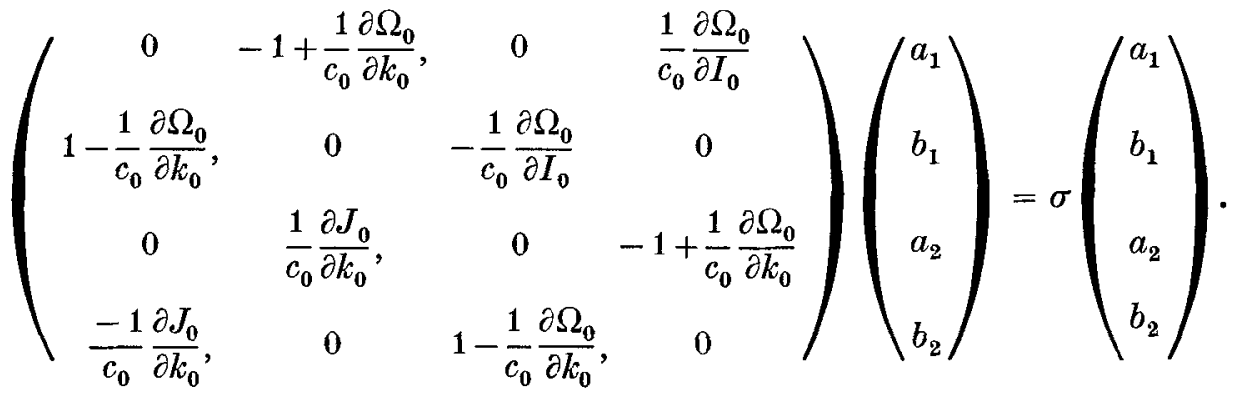

The eigenvalues are

$$
\sigma= \pm i\left(1-\frac{1}{c_{0}} \frac{\partial \Omega_{0}}{\partial k_{0}}\right) \pm\left(-\frac{\partial \Omega_{0}}{\partial I_{0}} \frac{\partial J_{0}}{\partial k_{0}}\right)^{\frac{1}{2}}
$$

It is known that the second term in (6.8) is real if the amplitude of the regular wave train is small, so that a uniform wave train is unstable. The instability is called the Benjamin-Feir instability. Peregrine \& Thomas (1979) using accurate computed properties of the waves showed that the second term vanished for $h / \lambda=0 \cdot 1093$ and becomes pure imaginary at greater values. Thus the Benjamin-Feir instability, for infinitely long modulations, vanishes for waves of large amplitude. This was predicted by Lighthill (1967) using an approximate Lagrangian to occur at $h / \lambda=0 \cdot 108$. Note that the exchange of stability is not associated with neutral stability.

It follows from (6.3) and (6.4) after some algebra that

$$
-\frac{\partial \Omega}{\partial I} \frac{\partial J}{\partial k}=\left(\frac{13}{2} T-6 V\right) \frac{\partial \Omega}{\partial E}-25 T^{2}\left(\frac{\partial \Omega}{\partial E}\right)^{2}
$$

A point of neutral stability occurs when (6.9) is negative and

$$
\pm\left[25 T^{2}\left(\frac{\partial \Omega}{\partial E}\right)^{2}-\left(\frac{13}{2} T-6 V\right) \frac{\partial \Omega}{\partial E}\right]^{\frac{1}{2}}=1-\frac{1}{c} \frac{\partial \Omega}{\partial k}=\frac{1}{2}-2 E \frac{\partial \Omega}{\partial E}
$$

The smallest wave for which this equation is satisfied is $h / \lambda=0.1301$ (it occurs with the positive sign). It is obvious that this point of neutral stability is not an exchange 
of stability. It will now be shown that it is also not a bifurcation point. Note, incidentally, that this value $h / \lambda$ is greater than the critical values shown in table 1 .

The simplest approach is to use the fact that a steady solution must have the form

$$
k=f(x-U t), \quad I=g(x-U t) .
$$

Then (6.1) and (6.2) can be integrated to give

$$
-U k+\Omega=0, \quad-U I+J=B
$$

where $B$ is an arbitrary constant of integration. In this equation, $\Omega$ and $J$ are given functions of $k$ and $I$. These equations imply $k=$ constant, $I=$ constant; and the solution is a uniform wave. Equations (6.12) define $I$ and $k$ as functions of $B$ and $U$. The critical condition given by $(6.10)$ expresses the result that the relation is not $1-1$ in the neighbourhood of values satisfying $(6.10)$, but non-constant solutions do not exist.

The computations were carried out on the CDC STAR 100 at the CDC Service Center, Minneapolis. The provision of time on the machine by Control Data Corporation is gratefully acknowledged. Dr H. C. Yuen is thanked for the benefit of many stimulating discussions. The research was supported by the Office of Naval Research.

\section{REFERENCES}

Chen, B. \& Saffman, P. G. 1980 Numerical evidence for the existence of new types of gravity wave of permanent form on deep water. Stud. Appl. Math. 62, 1-21.

Cokelet, E. D. 1974 Steep gravity waves in water of arbitrary uniform depth. Phil. Trans. Roy. Soc. A 286, 183-230.

Garabedian, P. R. 1965 Surface waves of finite depth. J. D'Analyse Math. 14, 161-169.

Keller, H. B. 1977 Numerical solution of bifurcation and nonlinear eigenvalue problems. Applications of Bifurcation Theory, pp. 359-384. Academic.

Lighthill, M. J. 1967 Some special cases treated by the Whitham theory. Proc. Roy. Soc. A 299, 28-53.

LoNGUET-Hragins, M.S. 1975 Integral properties of periodic gravity waves of finite amplitude. Proc. Roy. Soc. A 342, 157-174.

Longuet-Higarns, M. S. 1978 a Some new relations between Stokes' coefficients in the theory of gravity waves. J. Inst. Math. Applics. 22, 261-273.

Longuet-Hrgains, M. S. $1978 b$ The instabilities of gravity waves of finite amplitude in deep water. II. Subharmonics. Proc. Roy. Soc. A 360, 489-505.

Peregrine, D. H. \& Thomas, G. P. 1979 Finite amplitude deep water waves on currents. Phil. Trans. Roy. A 292, 371-390.

Saffman, P. G. \& Szeto, R. 1980 Structure of a linear array of uniform vortices. Stud. Appl. Math. (to appear).

Schwartz, L. W. 1974 Computer extension and analytic continuation of Stokes expansion for gravity waves. $J$. Fluid Mech.62, 553-578.

Stokes, G. G. 1880 Supplement to a paper on the theory of oscillatory waves. Mathematical and Physical Papers, vol. 1, pp. 225-228. Cambridge University Press.

Whithaм, G. B. 1965 A general approach to linear and nonlinear dispersive waves using $a$ Lagrangian. J. Fluid Mech. 22, 273-283.

Whitham, G. B. 1970 Two-timing, variational principles and waves. J. Fluid Mech. 44, 373395.

Whiтнам, G. B. 1974 Linear and Non-linear waves. Wiley-Interscience. 